\title{
Classical Systems and Observables in Quantum Mechanics
}

\author{
Holger NeumanN \\ Institut für Theoretische Physik (I) der Universität Marburg (Lahn)
}

Received March 29, 1971

\section{Introduction}

In [1] Chap. III Ludwig gave the definition of coexistent effects in a quantum mechanical system. Coexistence of effects will be discussed in this paper. Special attention will be called to the connection between the notion "observable" and the notion "classical system". It will be proved in particular that the set of classical effects is coexistent and therefore the convex range of observables consists of coexistent effects.

We shall stick closely to the notation introduced in [1]. The quantum mechanical system is described by subsets of an ordered separable Banach space $B$ and its dual $B^{\prime}$, which satisfy several axioms. The subset

$$
K=\{X / X \in B, X \geqq 0,\|X\|=1\}
$$

of $B$ represents the ensembles, and the subset

$$
\hat{L}=\left\{Y / Y \in B^{\prime}, Y \geqq 0,\|Y\| \leqq 1\right\}
$$

of $B^{\prime}$ represents the effects of the system (yes-no-experiments). The probability to measure the effect $F \in \hat{L}$ in the ensemble $V \in K$ is expressed by the value $\langle V, F\rangle$ of the functional $F$ on $B$. $G$ denotes the set of extreme points of $\hat{L}$, the set of decision effects. $G$ is a complete, orthocomplemented, weakly modular lattice.

Perhaps it is convenient to keep in mind the representation of $B$ and $B^{\prime}$ by Hermitean operators in a separable Hilbert space. This representation is valid for irreducible quantum mechanical systems (no superselection rule).

$B$ is the set of Hermitean trace class operators.

$B^{\prime}$ is the set of bounded Hermitean operators.

( $\geqq$ is the usual order of Hermitean operators.)

$K=\{V \in B / V \geqq 0, \operatorname{tr}(V)=1\}, \hat{L}=\left\{F \in B^{\prime} / 0 \leqq F \leqq 1\right\}$.

$G$ is the lattice of projection operators.

$$
\langle V, F\rangle=\operatorname{tr}(V \cdot F)
$$




\section{Coexistent Effects}

\subsection{Definition of Coexistence}

Let $Q$ be a Boolean ring (Boolean lattice or Boolean algebra) with the lattice operations: $q_{1} \wedge q_{2}$ intersection, $q_{1} \vee q_{2}$ union, $q^{*}$ complement, $q_{1}+q_{2}=\left(q_{1} \wedge q_{2}^{*}\right) \vee\left(q_{1}^{*} \wedge q_{2}\right)$ symmetric difference. An $\hat{L}$-valued measure on $Q$ is a mapping $F: Q \rightarrow \hat{L}$ such that $F\left(q_{1} \vee q_{2}\right)=F\left(q_{1}\right)+F\left(q_{2}\right)$ if $q_{1} \wedge q_{2}=0$. The $\hat{L}$-valued measure $F(q)$ on $Q$ is said to be effective if $F(q)=0$ implies $q=0$.

Roughly spoken a subset $l \subset \hat{L}$ is said to be a set of coexistent effects if it is possible to construct an apparatus in which these effects can be measured together. By analyzing the physical situation Ludwig ([1] Chap. III, 12) gives the following mathematical translation of this fact:

Definition 1. A subset $l \subset \hat{L}$ is said to be a set of coexistent effects, if there is a Boolean ring $Q$ and an $\hat{L}$-valued measure $F(q)$ on $Q$ such that $l$ is contained in the range of $F(q)$.

Here $Q$ stands for the different signal-parts of the apparatus in which the effects are measured together. The Boolean operations in $Q$ correspond to technical operations with these signal-parts as follows:

The signal-part $q_{1} \wedge q_{2}$ is always excited if both $q_{1}$ and $q_{2}$ are excited.

The signal-part $q_{1}+q_{2}$ is always excited if either $q_{1}$ or $q_{2}$ (but not both) is excited.

$\left(q^{*}\right.$ and $q_{1} \vee q_{2}$ can be expressed by $q^{*}=1+q, q_{1} \vee q_{2}=q_{1}+q_{2}$ $\left.+\left(q_{1} \wedge q_{2}\right).\right)$

If $F(q)$ is an $\hat{L}$-valued measure on $Q$, the set $J=\{q / q \in Q, F(q)=0\}$ is an ideal in $Q$ and by passing from $Q$ to the quotient ring $Q / J$ it is possible to get an effective $\hat{L}$-valued measure without altering the range of $Q$.

Likewise it is possible to extend the Boolean ring $Q$ and the $\hat{L}$-valued measure such that $F(1)=1$, where the first 1 denotes the unit element in $Q$ and the second 1 denotes the order unit in $\hat{L} \subset B^{\prime}$. Thus we may confine ourselves in our considerations to the case where $F(q)$ is an effective $\hat{L}$-valued measure with $F(1)=1$.

\subsection{A Uniform Structure on $Q$}

An ensemble $V \in K$ is called effective if $\langle V, F\rangle=0, F \in \hat{L}$ implies $F=0$. As $B$ is assumed to be separable there exists an ensemble

$$
\begin{aligned}
& V_{0}=\sum_{v=1}^{\infty} \lambda_{v} \cdot V_{v}, \lambda_{v}>0, \quad \sum_{v=1}^{\infty} \lambda_{v}=1 \text { and } \\
& \left\{V_{v}\right\} \text { is a countable dense subset of } K .
\end{aligned}
$$


$V_{0}$ is an effective ensemble and $m_{0}(q)=\left\langle V_{0}, F(q)\right\rangle$ is an effective realvalued (not necessarily $\sigma$-additive) measure on $Q$.

Thus $d\left(q_{1}, q_{2}\right)=m_{0}\left(q_{1}+q_{2}\right)$ is a metric on $Q$ and the lattice operations are uniformly continuous with respect to this metric [2]. The uniform structure $u_{K}$ induced by this metric is determined by the neighbourhoods of the 0 -element of $Q$ :

$$
U_{V_{0}, \varepsilon}=\left\{q / q \in Q, m_{V_{0}}(q)=\left\langle V_{0}, F(q)\right\rangle \leqq \varepsilon\right\} .
$$

The uniform structure $u_{K}$ is independent of the choice of $V_{0}$ with property (1). We shall see this in proving that the filterbasis

$$
\begin{gathered}
U_{V_{1}, \ldots, V_{n}, \varepsilon}=\left\{q \in Q /\left\langle V_{i}, F(q)\right\rangle \leqq \varepsilon, i=1, \ldots, n\right\} \\
V_{1}, \ldots, V_{n} \text { varying in } K
\end{gathered}
$$

is equivalent to (2).

Of course (3) is finer than (2). A moment's thinking shows that the inverse is proved by the following inequality $\left(\left\{V_{v}\right\}\right.$ dense in $K$ !):

$$
\begin{aligned}
\langle V, F(q)\rangle & \leqq\left\langle V_{v}-V, F(q)\right\rangle \mid+\left\langle V_{v}, F(q)\right\rangle \\
& \leqq\left\|V_{v}-V\right\|+\lambda_{v}^{-1}\left\langle V_{0}, F(q)\right\rangle \text { for all } V \in K .
\end{aligned}
$$

As a basis of the uniform structure $u_{K}$ is given by the subsets

$$
\left\{\left(q_{1}, q_{2}\right) \in Q \times Q / q_{1}+q_{2} \in U_{V_{1}, \ldots, V_{n, \varepsilon}}\right\}
$$

of $Q \times Q$ and $q_{1}+q_{2}$ denotes the "difference" of the signal-parts $q_{1}$ and $q_{2}, u_{K}$ is the natural uniform structure induced by testing the signalparts of $Q$ by a finite number of ensembles.

Lemma 1. The mapping $F: Q \rightarrow \hat{L}$ of the $\hat{L}$-valued effective measure $F(q)$ is uniformly $u_{K}-\sigma\left(B^{\prime}, B\right)$-continuous.

Proof. From $q_{1}=\left(q_{1} \wedge q_{2}^{*}\right) \vee\left(q_{1} \wedge q_{2}\right) \leqq\left(q_{1}+q_{2}\right) \vee q_{2}$ follows $F\left(q_{1}\right)$ $\leqq F\left(q_{1}+q_{2}\right)+F\left(q_{2}\right)$ and $F\left(q_{2}\right) \leqq F\left(q_{1}+q_{2}\right)+F\left(q_{1}\right)$ likewise. Thus $\left|\left\langle V, F\left(q_{1}\right)-F\left(q_{2}\right)\right\rangle\right| \leqq\left\langle V, F\left(q_{1}+q_{2}\right)\right\rangle$ for all $V \in K$. As $X \in B$ can be decomposed in $X=\alpha V_{1}-\beta V_{2} ; V_{1}, V_{2} \in K([1] \mathrm{III}, 6)$ the $\sigma\left(B^{\prime}, B\right)$-topology is completely determined by the elements of $K$ (instead of $B$ ). The representation (3) of the basis of $u_{K}$ and the definition of the $\sigma\left(B^{\prime}, B\right)$ topology prove the statement.

Let $\tilde{Q}$ be the completion of $Q$ with respect to the uniform structure $u_{K}$ resp. metric $d\left(q_{1}, q_{2}\right)=m_{V_{0}}\left(q_{1}+q_{2}\right)$. Because of the uniform continuity of the lattice operations in $Q$ the lattice operations can be extended to the whole of $\tilde{Q}$ such that $\tilde{Q}$ is a Boolean ring. As $u_{K}$ is induced by a real-valued measure $m_{V_{0}}$, one may apply the results of measure theory on Boolean rings and conclude that $\tilde{Q}$ is a Boolean $\sigma$-ring (which is 
even lattice complete such that unions and intersections can be performed of all subsets of $\tilde{Q}$ ) [3].

Since the uniform structure $u_{K}$ on $Q$ has got a physical meaning and the elements of $\tilde{Q}$ are $u_{K}$-limit points of elements of $Q$, we may assign the same interpretation to elements of $\tilde{Q}$ as to the elements of $Q . u_{K}$ is used to describe the uncertainty of the correspondence between signalparts and elements of $Q$.

$\hat{L}$ is a $\sigma\left(B^{\prime}, B\right)$-closed subset of the unit sphere of $B^{\prime}$. Thus $\hat{L}$ is $\sigma\left(B^{\prime}, B\right)$ compact and complete with respect to the uniform structure determined by the $\sigma\left(B^{\prime}, B\right)$-topology. Therefore the uniform continuous mapping $F: Q \rightarrow \hat{L}$ has a unique extension to $\tilde{Q}$. Because of the uniqueness $F\left(q_{1} \vee q_{2}\right)$ $=F\left(q_{1}\right)+F\left(q_{2}\right)$ holds for all $q_{1}, q_{2} \in \tilde{Q}$ with $q_{1} \wedge q_{2}=0$. Thus $F(q)$ is extended to an $\hat{L}$-valued measure on $\tilde{Q}$, and we have proved

Theorem 1 (Ludwig). A subset $l \subset \hat{L}$ consists of coexistent effects if there exists a Boolean ring $Q$ and an effective $\hat{L}$-valued measure $F(q)$ on $Q$ with $F(1)=1$ such that $Q$ is $u_{\mathrm{K}}$-complete (and therefore a $\sigma$-ring which is even lattice complete), and $l$ is contained in the range of $F(q)$.

Corollary. Let $Q$ be a Boolean ring and $F(q)$ an effective $\hat{L}$-valued measure with $F(1)=1$ such that $Q$ is $u_{\mathrm{K}}$-complete. $m_{V}(q)=\langle V, F(q)\rangle$ is a real-valued normed $\left(m_{V}(1)=1\right) \sigma$-additive measure on $Q$ for all $V \in K$.

Proof. Let $q_{n} \in Q, q_{n+1} \leqq q_{n}$ and $\bigwedge_{n=1}^{\infty} q_{n}=0$. To prove the $\sigma$-additivity it suffices to show

$$
m_{V}\left(q_{n}\right) \rightarrow 0 \text { for all } V \in K .
$$

Taking into account the representation (3) of a basis of neighbourhoods of 0 we conclude that (4) is equivalent to $q_{n} \rightarrow 0$ with respect to the uniform structure $u_{K}$. Let $V_{0} \in K$ have property (1) such that $d\left(q_{1}, q_{2}\right)=m_{V_{0}}\left(q_{1}+q_{2}\right)$ $=\left\langle V_{0}, F\left(q_{1}+q_{2}\right)\right\rangle$ induces $u_{\mathbf{K}}$. As $m_{V_{0}}\left(q_{n}\right) \geqq 0$ is monotonely decreasing $m_{V_{0}}\left(q_{n}\right) \rightarrow \alpha \geqq 0$. From $m_{V_{0}}\left(q_{n}+q_{n^{\prime}}\right)=\left|m_{V_{0}}\left(q_{n}\right)-m_{V_{0}}\left(q_{n^{\prime}}\right)\right|$ follows that $q_{n}$ is a $u_{K}$-Cauchy-sequence and therefore converges to $q \in Q$ with respect to $u_{K}$. The continuity of the lattice operations implies $q \leqq q_{n}$, and from $\bigwedge_{n=1}^{\infty} q_{n}=0$ follows $q=0$.

As Ludwig pointed out in [1] Chap. II, 9, those sets of a mathematical theory which in a physical theory are in correspondence with objects of reality should be separable with respect to the uniform structure describing the uncertainty of this correspondence. This fact motivates the following definition:

Definition 2. A Boolean ring $Q$ together with an effective $\hat{L}$-valued measure $F(q)$ on $Q$ such that $F(1)=1$ and $Q$ is $u_{\mathrm{K}}$-complete and separable 
is said to be an observable $O=(Q, F(q))$. An observable $O=(Q, F(q))$ is called decision observable if the range of the $\hat{L}$-valued measure $F(q)$ consists of decision effects, i.e. $F(q) \in G$ for all $q \in Q$. ( $G$ is the lattice of extreme points of $\hat{L}$.)

In view of Theorem 1 the question arises whether it is possible to confine oneself to the consideration of observables, i.e. $u_{K}$-separable Boolean rings while dealing with coexistent effects. This question will be settled in Section 5 .

We shall not treat the problem of decision observables here. This is done in detail in [1]. It is shown there that the mapping $Q \rightarrow G \subset \hat{L}$ given by a decision observable $O=(Q, E(q))$ is a lattice isomorphism of $Q$ onto a Boolean sublattice of the lattice $G$ of decision effects. Thus it is possible to transcribe the physical interpretation of the lattice operations of $Q$ to the lattice operations in the Boolean sublattice of $G$.

In case of a decision observable the $u_{K}$-completeness of $Q$ is proved to be equivalent to the lattice completeness [1], and in theorem 7 we shall see, as a by-product, that the condition of $u_{\mathrm{K}}$-separability of $Q$ is automatically fulfilled. Thus a decision observable is a lattice complete Boolean sublattice of $G$.

\section{Classical Systems}

Throughout this chapter $Q$ denotes a Boolean $\sigma$-ring and $m_{0}$ an effective real-valued normed $\sigma$-additive measure on $Q . B_{Q}$ denotes the linear space of all $\sigma$-additive bounded real-valued functions on $Q$.

$B_{Q}=\left\{m(q) / m(q)\right.$ real, $|m(q)| \leqq C, m\left(\bigvee_{i=1}^{\infty} q_{i}\right)=\sum_{i=1}^{\infty} m\left(q_{i}\right)$

The total variation

$$
\text { for all } \left.\left\{q_{i}\right\} \text { with } q_{i} \wedge q_{k}=0 \text { if } i \neq k\right\} \text {. }
$$

$$
|m|(q)=\sup \left\{\lambda / \lambda=\sum_{i=1}^{n}\left|m\left(q_{i}\right)\right|, q=\bigvee_{i=1}^{n} q_{i}, q_{i} \wedge q_{k}=0 \text { if } i \neq k\right\}
$$

is a $\sigma$-additive measure on $Q$ and thus an element of $B$. It is well known that $B_{Q}$ is a Banach space with respect to the norm $\|m\|=|m|(1)$. As $m_{0}$ is effective on $Q$ all elements of $B_{Q}$ are absolutely continuous with respect to $m_{0}$.

If $(X, A, J)$ is a representation of $Q$ by a Boolean $\sigma$-ring $A$ of subsets of a set $X$ with a $\sigma$-ideal $J$ of $A$ such that $A / J$ is $\sigma$-isomorphic to $Q$ (for instance the Loomis' representation), the dual space $B_{Q}^{\prime}$ of $B_{Q}$ may be characterized by the space of all $m_{0}$-measurable bounded functions on $X$. 
In the sequel we want to sketch a representation - free characterization of $B_{Q}^{\prime}$.

A finite subset $\left\{q_{i}\right\}_{i=1 \ldots n} \subset Q$ is said to be a decomposition of 1 if $q_{i} \neq 0$ for all $i, q_{i} \wedge q_{k}=0$ if $i \neq k$ and $\bigvee_{i=1}^{n} q=1$

A mapping $f:\left\{q_{i}\right\} \rightarrow \mathbb{R}$ of a decomposition of 1 into the reals is said to be a representative of a simple function, and the set of these mappings is denoted by $\tilde{S}(Q)$.

Let $f, f^{\prime} \in \tilde{S}(Q)$, where $f:\left\{q_{i}\right\} \rightarrow \mathbb{R}$ and $f^{\prime}:\left\{q_{k}^{\prime}\right\} \rightarrow \mathbb{R}$.

The relation $f\left(q_{i}\right)=f^{\prime}\left(q_{k}^{\prime}\right)$ if $q_{i} \wedge q_{k}^{\prime} \neq 0$ is an equivalence relation $f \sim f^{\prime}$ on $\tilde{S}(Q)$.

$S(Q)$ denotes the set of equivalence classes, which will be called simple functions on $Q$.

It is easily seen that in every equivalence class there is one and only one representative $f \in \tilde{S}(Q)$, the values of which are different for different elements of the decomposition of 1 (the mapping $f$ is injective). The equivalence classes, simple functions, will be characterized by representatives.

$S(Q)$ is a linear vector space by means of the following definitions:

1. $f+f^{\prime}$ is a mapping of

$$
\left\{q_{i} \wedge q_{k}^{\prime} / q_{i} \in\left\{q_{i}\right\}, q_{k}^{\prime} \in\left\{q_{k}^{\prime}\right\}, q_{i} \wedge q_{k}^{\prime} \neq 0\right\}
$$

into $\mathbb{R}$ such that $\left(f+f^{\prime}\right)\left(q_{i} \wedge q_{k}^{\prime}\right)=f\left(q_{i}\right)+f\left(q_{k}^{\prime}\right)$.

2. $\lambda \cdot f$ is a mapping of $\left\{q_{i}\right\}$ into $\mathbb{R}$ such that $(\lambda \cdot f)\left(q_{i}\right)=\lambda \cdot f\left(q_{i}\right), \lambda \in \mathbb{R}$. $\eta_{q}, q \in Q$, denotes the simple function $\eta_{q}\left\{q, q^{*}\right\} \rightarrow \mathbb{R}$ such that $\eta_{q}(q)=1$ and $\eta_{q}\left(q^{*}\right)=0$ and is said to be a characteristic function. Every simple function $f:\left\{q_{i}\right\} \rightarrow \mathbb{R}$ then has the representation $f=\sum_{i=1}^{n} f\left(q_{i}\right) \eta_{q_{i}}$.

$\langle m, f\rangle=\sum_{i=1}^{n} f\left(q_{i}\right) \cdot m\left(q_{i}\right), m \in B_{Q}, f \in S(Q)$ is a bilinear function on $B_{Q} \times S(Q)$ with the property

$$
\begin{aligned}
& \langle m, f\rangle=0 \text { for all } f \in S(Q) \text { implies } m=0, \\
& \langle m, f\rangle=0 \text { for all } m \in B_{Q} \text { implies } f=0 .
\end{aligned}
$$

As

$$
|\langle m, f\rangle| \leqq|m|(1) \cdot \sup _{i}\left|f\left(q_{i}\right)\right|
$$

holds, the linear functional on $B_{Q}$ given by $f \in S(Q)$ is continuous, and thus $S(Q)$ is a linear subspace of $B_{Q}^{\prime}$, the dual space of $B_{Q}$. Concerning 
the dual norm on $S(Q)$ we have

$$
\begin{aligned}
\|f\| & =\sup _{m \in B_{Q}}\left(\frac{1}{|m|(1)}|\langle m, f\rangle|\right) \\
& \geqq \sup _{q \in Q}\left(\frac{1}{m_{0 q}(1)}\left|\sum_{i=1}^{n} f\left(q_{i}\right) \cdot m_{0}\left(q \wedge q_{i}\right)\right|\right) \\
& \geqq \sup _{q \in Q}\left(\sup _{i}\left(\left|f\left(q_{i}\right)\right| \frac{m_{0}\left(q \wedge q_{i}\right)}{m_{0}(q)}\right)\right) \\
& =\sup _{i}\left|f\left(q_{i}\right)\right| .
\end{aligned}
$$

Here $m_{0 q}$ denotes the measure defined by $m_{0 q}\left(q^{\prime}\right)=m_{0}\left(q \wedge q^{\prime}\right)$. (5) and (6) imply

$$
\|f\|=\sup _{i}\left|f\left(q_{i}\right)\right| .
$$

It is possible to show that $S(Q)$ is dense in $B_{Q}^{\prime}$ with respect to the dual norm. Thus $B_{Q}^{\prime}$ may be identified with the completion of $S(Q)$ with respect to the norm (7).

The definition $f \geqq 0$ if $\langle m, f\rangle \geqq 0$ for all $m \in B_{Q}$ with $m \geqq 0$ gives the dual order in $B_{Q}^{\prime}$. If $f \in S(Q), f=\sum_{i=1}^{n} f\left(q_{i}\right) \eta_{q_{i}}$ one has $f \geqq 0$ if and only if $f\left(q_{i}\right) \geqq 0$ for all $i$.

In connection with the remarks at the end of Section 2.2 we shall now prove

Theorem 2. Let $Q, m_{0}, B_{Q}$ be as stated above. $B_{Q}$ is a separable Banach space if and only if $Q$ is separable with respect to the uniform structure $u_{K}$ which is determined by the metric $d\left(q_{1}, q_{2}\right)=m_{0}\left(q_{1}+q_{2}\right)$.

Proof. As $m_{0 q_{1}}-m_{0 q_{2}}=m_{0\left(q_{1} \wedge q_{2}^{\star}\right)}-m_{0\left(q_{1}^{\star} \wedge q_{2}\right)}$ holds for $q_{1}, q_{2} \in Q$, the total variation is

$$
\left|m_{0 q_{1}}-m_{0 q_{2}}\right|=m_{0\left(q_{1} \wedge q_{2}^{*}\right)}+m_{0\left(q_{1}^{*} \wedge q_{2}\right)}=m_{0\left(q_{1}+q_{2}\right)} .
$$

This implies

$$
\left\|m_{0 q_{1}}-m_{0 q_{2}}\right\|=m_{0}\left(q_{1}+q_{2}\right) .
$$

(8) shows that the subset $M=\left\{m_{0_{q}}, q \in Q\right\}$ of $B_{Q}$ and $Q$ are homeomorphic and $Q$ is $u_{K}$-separable if and only if $M$ is norm-separable.

First assume $B_{Q}$ to be separable. As $B_{Q}$ is a metric space the subset $M$ of $B_{Q}$ is separable.

Now assume $Q$ to be separable and $M_{C}$ a countable dense subset of $M$. Let

$$
T=\left\{m \in B_{Q} / m=\sum_{i=1}^{n} \lambda_{i} m_{0 q_{i}}, \lambda_{i} \text { real, } q_{i} \in Q\right\}
$$


$T$ is a linear subspace of $B_{Q}$. The Radon-Nikodym-theorem which establishes an isomorphism between $B_{Q}$ and the space of $m_{0}$-integrable functions $L_{1}\left(Q, m_{0}\right)$ shows that $T$ is dense in $B_{Q}$ corresponding to the simple functions $S(Q)$ in $L_{1}\left(Q, m_{0}\right)$.

$$
T_{C}=\left\{m \in B_{Q} / m=\sum_{i=1}^{n} \varrho_{i} \cdot m_{0 q_{i}}, \varrho_{i} \text { rational, } m_{0 q_{2}} \in M_{C}\right\}
$$

is a countable dense subset of $T$ and thus of $B_{Q}$.

In [1] Chap. III, 16 a system $\left\langle B, B^{\prime}\right\rangle$ as described in our introduction is said to be classical if the lattice $G$ of decision effects is Boolean (thus $G$ is a Boolean ring). It is shown that in this case $\left\langle B, B^{\prime}\right\rangle$ is isomorphic to $\left\langle B_{Q}, B_{Q}^{\prime}\right\rangle$ with $Q=G$ such that $K_{Q}=\left\{m \in B_{Q} / m \geqq 0, m(1)=1\right\}$ represents the ensembles, $\hat{L}_{Q}=\left\{f \in B_{Q}^{\prime} / 0 \leqq f \leqq 1\right\}$ represents the effects, and $G_{Q}=\left\{f \in \hat{L}_{Q}, f=\eta_{q}, q \in Q\right\}$ represents the decision effects of the system.

As $B$ is assumed to be separable theorem 2 shows that $Q$ is $u_{K}$-separable.

In the reverse given a Boolean $\sigma$-ring $Q$ with an effective measure $m_{0}$ such that $Q$ is $u_{K}$-separable a system $\left\langle B_{Q}, B_{Q}^{\prime}\right\rangle$ satisfies Ludwig's axioms and is a classical system.

\section{Observables and Classical Systems}

In this section it will be shown that an $\hat{L}$-valued measure $F(q)$ on a Boolean ring $Q$ which is $u_{\mathrm{K}}$-complete induces a linear mapping $S: B \rightarrow B_{Q}$. This mapping makes possible some interesting results with respect to coexistent effects which will be presented in Section 5.

We'll first give a definition [4].

Definition 3. Let $\left\langle B_{1}, B_{1}^{\prime}\right\rangle$ and $\left\langle B_{2}, B_{2}^{\prime}\right\rangle$ describe quantum mechanical systems, and let $K_{1}, K_{2}, \hat{L}_{1}, \hat{L}_{2}$ be the sets of ensembles and effects respectively. A mapping $S: K_{1} \rightarrow K_{2}$ which is linear on $K_{1}$ (i.e. $S\left(\mu V_{1}\right.$ $\left.\left.+(1-\mu) V_{2}\right)=S V_{1}+(1-\mu) S V_{2}, 0 \leqq \mu \leqq 1\right)$ is said to be a mixture homomorphism. A mapping $T: \hat{L}_{2} \rightarrow \hat{L}_{1}$ is said to be a continuous linear homomorphism if the following conditions are satisfied:

1. $F_{1}, F_{2}, F_{1}+F_{2} \in \hat{L}_{2}$ implies $T\left(F_{1}+F_{2}\right)=T F_{1}+T F_{2}$.

2. If $F_{n} \in \hat{L}_{2}$ is a $\sigma\left(B_{2}^{\prime}, B_{2}\right)$-convergent sequence $F_{n} \rightarrow F \in \hat{L}_{2}$ then $T F_{n} \sigma\left(B_{1}^{\prime}, B_{1}\right)$-converges to $T F$.

The following lemma gives a physical motivation of assumption 1 . of the second part of the definition.

Lemma 2. Assumption 1. of the second part of Definition 3 is equivalent to

$1^{\prime}$. If $F(q)$ is an $\hat{L}_{2}$-valued measure on a Boolean ring $Q, T F(q)$ is an $\hat{L}_{1}$-valued measure on $Q$. 
Proof. Trivially 1 implies $1^{\prime}$. In order to show the reverse suppose $F_{1}, F_{2}, F_{1}+F_{2} \in \hat{L}_{2}$. Consider the Boolean ring $Q(2)$ of all subsets of a set of two elements $\{1,2\}$ and define $F(\{\emptyset\})=0, F(\{1\})=F_{1}, F(\{2\})=F_{2}$, $F(\{1,2\})=F_{1}+F_{2} . F(q), q \in Q(2)$ is an $\hat{L}_{2}$-valued measure on $Q(2)$ and $T\left(F_{1}+F_{2}\right)=T(F(\{1\} \vee\{2\}))=T\left(F_{1}\right)+T\left(F_{2}\right)$.

As it is easily seen a mixture homomorphism has a unique linear extension to the whole of $B_{1}$. The extended linear mapping is positive and satisfies $\|S X\| \leqq\|X\|$ for all $X \in B_{1}$. Therefore the adjoint linear mapping $S^{\prime}: B_{2}^{\prime} \rightarrow B_{1}^{\prime}$ is $\sigma\left(B_{2}^{\prime}, B_{2}\right)-\sigma\left(B_{1}^{\prime}, B_{1}\right)$-continuous, positive and satisfies $\left\|S^{\prime} Y\right\| \leqq\|Y\|$ for all $Y \in B_{2}^{\prime}$. Moreover $S^{\prime} 1=1$ since $S K_{1} \subset K_{2}$.

A continuous linear homomorphism $T: \hat{L}_{2} \rightarrow \hat{L}_{1}$ has a unique linear extension to the whole of $B_{2}^{\prime}$. The extended linear mapping $T: B_{2}^{\prime} \rightarrow B_{1}^{\prime}$ is $\sigma\left(B_{2}^{\prime}, B_{2}\right)-\sigma\left(B_{1}^{\prime}, B_{1}\right)$-continuous, positive and satisfies $\|T Y\| \leqq\|Y\|$ for all $Y \in B^{\prime}$. Therefore the adjoint linear mapping $T^{\prime}$ maps $B_{1}$ into $B_{2}$, is positive and satisfies $\left\|T^{\prime} X\right\| \geqq\|X\|$ for all $X \in B_{1}$. If $T 1=1, T^{\prime} K_{1} \subset K_{2}$ holds, and $T^{\prime}$ is a linear extension of a mixture homomorphism. Thus the mixture homomorphisms of ensembles and the continuous linear homomorphisms of effects which map 1 onto 1 , are dually connected.

Theorem 4. Let $\left\langle B, B^{\prime}\right\rangle$ describe a quantum mechanical system. Let $Q$ be a Boolean ring and $F(q)$ an $\hat{L}$-valued effective measure such that $F(1)=1$ and $Q$ is $u_{K^{-}}$complete.

Define $S V=m_{V}$ for $V \in K$, where $m_{V}(q)=\langle V, F(q)\rangle$ is as in the corollary of theorem 1. Then it is defined a mixture homomorphism $S: K \rightarrow K_{Q}$, which maps effective ensembles onto effective measures and the continuous linear homomorphism $S^{\prime}: \hat{L}_{Q} \rightarrow \hat{L}$ defined by the adjoint $S^{\prime}$ of the linear extension of $S$ satisfies

$$
S^{\prime} \eta_{q}=F(q) \text { for all } q \in Q
$$

$S$ is uniquely determined by (9).

Proof. The corollary of Theorem 1 shows that indeed $S V=m_{V}$ defines a mapping $S: K \rightarrow K_{Q}$. The linearity of the function $\langle V, F(q)\rangle$ on $B$ implies that $S$ is a mixture homomorphism.

Assume $V_{0}$ to be an effective ensemble and $m_{V_{0}}(q)=0$. It follows $F(q)=0$ and $q=0$.

The following identity proves $S^{\prime} \eta_{q}=F(q)$

$$
V \in K,\left\langle V, S^{\prime} \eta_{q}\right\rangle=\left\langle S V, \eta_{q}\right\rangle=\left\langle m_{V}, \eta_{q}\right\rangle=m_{V}(q)=\langle V, F(q)\rangle .
$$

Now assume $\left\langle\left(S_{1}-S_{2}\right), \eta_{q}\right\rangle=0$ for all $q \in Q$.

It follows $\left\langle\left(S_{1}-S_{2}\right) V, f\right\rangle=0$ for all elements $f$ of the $\sigma\left(B_{Q}^{\prime}, B_{Q}\right)$ closure of the linear subspace $S(Q)$ of $B_{Q}^{\prime}$ spanned by the characteristic functions $\eta_{q}$. As $S(Q)$ is dense in $B_{Q}^{\prime}$ even with respect to the dual norm, $\left(S_{1}-S_{2}\right) V=0$ holds for all $V \in K$. 
It is possible to prove the inversion of Theorem 4:

Theorem 5. Let $Q$ be a Boolean $\sigma$-ring, $\left\langle B_{Q}, B_{Q}^{\prime}\right\rangle$ as described in section 2 and $S: K \rightarrow K_{Q}$ a mixture homomorphism which maps effective ensembles onto effective measures ${ }^{1}$. The adjoint continuous linear homomorphism $S^{\prime}: \hat{L}_{Q} \rightarrow \hat{L}$ defines an effective $\hat{L}$-valued measure $F(q)=S^{\prime} \eta_{q}$ such that $F(1)=1$ and $Q$ is $u_{\mathrm{K}}$-complete.

Proof. As $\eta_{q_{1} \vee q_{2}}=\eta_{q_{1}}+\eta_{q_{2}}$ if $q_{1} \wedge q_{2}=0, F(q)$ is an $\hat{L}$-valued measure.

$$
m_{V}(q)=\langle V, F(q)\rangle=\left\langle S V, \eta_{q}\right\rangle=(S V)(q) \text { proves } S V=m_{V} \text {. }
$$

To prove that $F(q)$ is effective suppose $F(q)=0$. Hence $m_{V_{0}}(q)=\left\langle V_{0}\right.$, $F(q)\rangle=0$ for an effective ensemble $V_{0} \in K$. Since $S^{\prime}(1)=1$ for an adjoint mapping of a mixture homomorphism, $F(1)=1$ holds.

To show the $u_{K}$-completeness of $Q$ let $V_{0}$ be an effective ensemble with property (1) of Section 1 . The uniform structure $u_{K}$ is generated by the metric $d\left(q_{1}, q_{2}\right)=m_{V_{0}}\left(q_{1}+q_{2}\right)$. As $m_{V_{0}}$ is an effective $\sigma$-additive measure on a Boolean $\sigma$-ring $Q, Q$ is complete with respect to this metric [2].

The combination of Theorem 2 with Theorems 4 and 5 gives

Corollary. To an observable $O=(Q, F(q))$ uniquely corresponds a mixture homomorphism $S$ of the quantum mechanical ensembles $K$ into the classical ensembles $K_{Q}, S: K \rightarrow K_{Q}$ such that $S$ maps effective ensembles on effective measures and $S^{\prime} \eta_{q}=F(q)$.

Vice versa a mixture homomorphism $S: K \rightarrow K_{Q}$ of the quantum mechanical ensembles $K$ into the classical ensembles $K_{Q}$ of a classical system, such that $S$ maps effective ensembles on effective measures, determines an observable $O=\left(Q, F(q)=S^{\prime} \eta_{q}\right)$.

\section{The Convex Range of Observables}

If $l$ is a set of effects of a quantum mechanical system described by $\left\langle B, B^{\prime}\right\rangle$, the $\sigma\left(B^{\prime}, B\right)$-closed convex hull of $l$ will be denoted by $\overline{c o}(l)$. Let $Q$ be a Boolean ring and $F(q)$ an $\hat{L}$-valued measure on $Q . \overline{c o}\{F(q)$, $q \in Q\}$ is called the convex range of the $\hat{L}$-valued measure. Convex combinations of effects $F(q)$ have a distinct physical meaning. If $F=\sum_{i=1}^{n} \lambda_{i} \cdot F\left(q_{i}\right), \lambda_{i}>0, \sum_{i=1}^{n} \lambda_{i}=1, F$ may be interpreted to be an effect of an apparatus, which selects the signal-parts $q_{i}$ with a frequency $\lambda_{i}$ and gives a response if the selected $q_{i}$ gives a response. A trivial example is given by the position measurement of an interval the endpoints of which are varied statistically.

\footnotetext{
1 This is no essential assumption but can always be arranged by altering the Boolean
} $\sigma$-ring $Q$ in correspondence with the effectivity of the $\hat{L}$-valued measure $F(q)$. 
Since coexistence of effects expresses the fact that these effects can be measured together in one apparatus the above given interpretation of convex combinations of effects suggests the conjecture that convex combinations of coexistent effects are coexistent. Indeed it will be proved by Theorem 8 that the convex range of an $\hat{L}$-valued measure consists of coexistent effects.

Definition 4. Let $Q, Q^{\prime}$ be Boolean rings and $F(q), F^{\prime}\left(q^{\prime}\right) \hat{L}$-valued measures on $Q$ and $Q^{\prime}$ respectively. $F(q)$ and $F^{\prime}\left(q^{\prime}\right)$ are said to be equivalent if

$$
\overline{\mathrm{co}}\{F(q), q \in Q\}=\overline{\mathrm{co}}\left\{F^{\prime}\left(q^{\prime}\right), q^{\prime} \in Q^{\prime}\right\} .
$$

Theorem 6. Let $Q$ be a Boolean ring and $F(q)$ an effective $\hat{L}$-valued measure on $Q$ such that $F(1)=1$ and $Q$ is $u_{K^{-}}$complete. Let $S: K \rightarrow K_{Q}$ be the mixture homomorphism determined by $F(q)$ corresponding to Theorem 4 and $S^{\prime}$ the adjoint continuous linear homomorphism. $S^{\prime}: \hat{L}_{Q} \rightarrow \hat{L}$.

1. $S^{\prime}\left(\hat{L}_{Q}\right)=\overline{\mathrm{co}}\{F(q), q \in Q\}$.

2. For every extreme point $F_{e}$ of $S^{\prime}\left(\hat{L}_{Q}\right)$ there is exactly one $q \in Q$ such that $F_{e}=F(q)$.

3. $\overline{\mathrm{co}}\{F(q), q \in Q\} \cap G=\{F(q), q \in Q\} \cap G$.

(It is not possible to get any new decision effects as limit points in the convex range of an $\hat{L}$-valued measure.)

Proof. The set of extreme points of $\hat{L}_{Q}$ is the set of characteristic functions $\left\{\eta_{q}, q \in Q\right\}$. Since $\hat{L}_{Q}$ is $\sigma\left(B_{Q}^{\prime}, B_{Q}\right)$-compact the Krein-Milmantheorem shows $\hat{L}_{Q}=\overline{\mathrm{co}}\left\{\eta_{q}, q \in Q\right\}$.

In Theorem 4 we proved $S^{\prime} \eta_{q}=F(q)$. Thus we have

$$
S^{\prime}\left(\operatorname{co}\left\{\eta_{q}, q \in Q\right\}\right)=\operatorname{co}\{F(q), q \in Q\} .
$$

The weak continuity of $S^{\prime}$ implies

$$
S^{\prime}\left(\hat{L}_{Q}\right) \subset \overline{\operatorname{co}}\{F(q), q \in Q\} .
$$

Since $\hat{L}_{Q}$ is $\sigma\left(B_{Q}^{\prime}, B_{Q}\right)$-compact $S^{\prime}\left(\hat{L}_{Q}\right)$ is $\sigma\left(B^{\prime}, B\right)$-compact and therefore closed such that

$$
S^{\prime}\left(\hat{L}_{Q}\right)=\overline{\operatorname{co}}\{F(q), q \in Q\} .
$$

Now assume $F_{e}$ to be an extreme point of $S^{\prime}\left(\hat{L}_{Q}\right)$. If $f \in \hat{L}_{Q}$ with $S^{\prime} f=F_{e}$, $f$ is an extreme point of $\hat{L}_{Q}$. Thus there is $q \in Q$ such that $F_{e}=F(q)$. Suppose $F_{e}=F\left(q_{1}\right)=F\left(q_{2}\right)$

$$
\begin{aligned}
& F_{e}=F\left(q_{1}\right)=F\left(q_{1} \wedge q_{2}^{*}\right)+F\left(q_{1} \wedge q_{2}\right), \\
& F_{e}=F\left(q_{2}\right)=F\left(q_{2} \wedge q_{1}^{*}\right)+F\left(q_{1} \wedge q_{2}\right) .
\end{aligned}
$$


Hence

$$
\begin{aligned}
1 / 2 F\left(q_{1}\right. & \left.\vee q_{2}\right)+1 / 2 F\left(q_{1} \wedge q_{2}\right) \\
& =1 / 2\left(F\left(q_{1} \wedge q_{2}^{*}\right)+F\left(q_{2} \wedge q_{1}^{*}\right)+F\left(q_{1} \wedge q_{2}\right)\right)+1 / 2 F\left(q_{1} \wedge q_{2}\right)=F_{e} .
\end{aligned}
$$

Since $F_{e}$ is an extreme point $F\left(q_{1} \vee q_{2}\right)=F\left(q_{1} \wedge q_{2}\right)=F_{e}$. Thus $F\left(q_{1}+q_{2}\right)$ $=F\left(q_{1} \vee q_{2}\right)-F\left(q_{1} \wedge q_{2}\right)=0$ and $q_{1}=q_{2}$ because of the effectivity of the $\hat{L}$-valued measure $F(q)$.

$S^{\prime}\left(\hat{L}_{Q}\right) \cap G=\{F(q), q \in Q\} \cap G$ is an immediate consequence as $G$ is the set of extreme points of $\hat{L}$ and $F \in S^{\prime}\left(\hat{L}_{Q}\right) \cap G$ thus implies $F$ is extreme point of $S^{\prime}\left(\hat{L}_{Q}\right)$.

Lemma 3. Let $Q$ be a Boolean ring and $F(q)$ an effective $\hat{L}$-valued measure on $Q$ such that $F(1)=1$ and $Q$ is $u_{K}$-complete. If $E$ denotes the set of extreme points of $\overline{\mathrm{co}}\{F(q), q \in Q\}$, the set $M=\{q \in Q, F(q) \in E\}$ is a $u_{K}$ separable subset of $Q$.

Proof. Theorem 6 shows that $F: Q \rightarrow \hat{L}$ gives a bijection of $M$ onto $E$. Since $B$ is separable, $\sigma\left(B^{\prime}, B\right)$ is metrizable in $\hat{L}$ and $\hat{L}$ is $\sigma\left(B^{\prime}, B\right)$-separable. Thus the subset $E \subset \hat{L}$ is $\sigma\left(B^{\prime}, B\right)$-separable and there is a countable $\sigma\left(B^{\prime}, B\right)$-dense subset $E_{C}$ in $E$. It will be shown that $M_{C}=F^{-1}\left(E_{C}\right)$ is $u_{K}$-dense in $M$.

If $q_{0} \in M$, there is a sequence $q_{n} \in M_{C}$ such that $F\left(q_{n}\right) \rightarrow F\left(q_{0}\right)$ in $\sigma\left(B^{\prime}, B\right)$.

$$
F\left(q_{n}\right)=F\left(q_{n} \wedge q_{0}\right)+F\left(q_{n} \wedge q_{0}^{*}\right) .
$$

As $\overline{c o}\{F(q), q \in Q\}$ is compact there is a subsequence $q_{n_{v}}$ such that

and

$$
F\left(q_{n_{v}} \wedge q_{0}\right) \rightarrow F_{1} \in \overline{\mathrm{co}}\{F(q), q \in Q\}
$$

$$
\begin{gathered}
F\left(q_{n_{v}} \wedge q_{0}^{*}\right) \rightarrow F_{2} \in \overline{\mathrm{co}}\{F(q), q \in Q\}, \\
F_{1}+F_{2}=F\left(q_{0}\right) .
\end{gathered}
$$

It suffices to show $q_{n_{v}} \rightarrow q_{0}$ with respect to $u_{k}$ or equivalently

$$
d\left(q_{n_{v}}, q_{0}\right)=m_{V_{0}}\left(q_{n_{v}}+q_{0}\right)=\left\langle V_{0}, F\left(q_{n_{v}}+q_{0}\right)\right\rangle \rightarrow 0 .
$$

$V_{0}$ being an effective ensemble with property (1) of Section 1. As

$$
\left\langle V_{0}, F\left(q_{n_{v}}+q_{0}\right)\right\rangle=\left\langle V_{0}, F\left(q_{n_{v}} \wedge q_{0}^{*}\right)\right\rangle+\left\langle V_{0}, F\left(q_{n_{v}}^{*} \wedge q_{0}\right)\right\rangle
$$

and $F\left(q_{n_{v}}^{*} \wedge q_{0}\right)=F\left(q_{0}\right)-F\left(q_{n_{v}} \wedge q_{0}\right)$, it suffices to show $F_{2}=0$ in Eq. (10). Since $F\left(q_{n_{v}} \vee q_{0}\right)=F\left(q_{n_{v}} \wedge q_{0}^{*}\right)+F\left(q_{0}\right) \rightarrow F_{2}+F\left(q_{0}\right)$ we have $F_{2}+F\left(q_{0}\right)$ $\in \overline{\mathrm{co}}\{F(q), q \in Q\} . F\left(q_{0}\right)=1 / 2 F_{1}+1 / 2\left(F_{2}+F\left(q_{0}\right)\right)$ implies $F_{1}=F_{2}+F\left(q_{0}\right)$ $=F\left(q_{0}\right)$, because $F\left(q_{0}\right)$ is an extreme point of $\overline{c o}\{F(q), q \in Q\}$. 
Theorem 7. Let $Q$ be a Boolean ring and $F(q)$ an effective $\hat{L}$-valued measure on $Q$ such that $F(1)=1$ and $Q$ is $u_{K}$-complete. There is a smallest Boolean subring $Q_{1}$ of $Q$ such that $Q_{1}$ is $u_{K}$-complete and the restriction $F_{1}(q)$ of $F(q)$ to $Q_{1}$ is equivalent to $F(q)$, i.e. $\overline{\mathrm{co}}\{F(q), q \in Q\}=\overline{\mathrm{co}}\left\{F_{1}(q)\right.$, $\left.q \in Q_{1}\right\} . Q_{1}$ is $u_{K^{-}}$-separable. Moreover $F(q) \in G$ for all $q \in Q$ implies $Q=Q_{1}$.

Proof. Let $E$ and $M$ be defined as in Lemma 3, $M_{C}$ a countable dense subset of $M$. The subring $Q_{C}$ of $Q$ generated by $M_{C}$ is countable. The closure $Q_{1}=\tilde{Q}_{c}$ with respect to $u_{K}$ is a $u_{K}$-separable and $u_{K}$-complete subring of $Q$. Clearly $M \subset Q_{1}$ and therefore $E$ is contained in the range of the restriction $F_{1}(q)$ of $F(q)$ to $Q_{1}$. Thus

$$
\overline{\mathrm{CO}}\{F(q), q \in Q\}=\overline{\mathrm{CO}}(E) \subset \overline{\mathrm{CO}}\left\{F_{1}(q), q \in Q_{1}\right\} .
$$

Of course $\overline{\mathrm{co}}\left\{F_{1}(q), q \in Q_{1}\right\} \subset \overline{\mathrm{co}}\{F(q), q \in Q\}$ and $\left(Q_{1}, F_{1}(q)\right)$ is equivalent to $(Q, F(q))$.

Suppose $Q^{\prime}$ is a $u_{K}$-complete Boolean subring of $Q$ such that the restriction of $F(q)$ to $Q^{\prime}$ is equivalent to $F(q)$. By Theorem 6, 2. we have $M \subset Q^{\prime}$ and thus $Q_{1} \subset Q^{\prime}$.

If $F(q) \in G, F(q)$ is an extreme point of $\overline{c o}\{F(q), q \in Q\}$ and therefore $F(q) \in E$. Thus $F(q) \in G$ for all $q \in Q$ implies $Q \subset M \subset Q_{1}$, i.e. $Q=Q_{1}$.

Theorem 8. Let $Q$ be a Boolean $\sigma$-ring and $\left\langle B_{Q}, B_{Q}^{\prime}\right\rangle$ a system as described in Section 2. There is a Boolean ring $Q^{\prime}$ such that $Q$ is a subring of $Q^{\prime}$, the $\hat{L}_{Q^{-v a l u e d}}$ measure $q \rightarrow \eta_{q}$ can be extended to $Q^{\prime}$ and the range of the extended $\hat{L}_{Q}$-valued measure consists of the entire set $\hat{L}_{Q}$.

Before we enter into the proof of Theorem 8 it will be shown that it is possible to define a multiplication in $B_{Q}^{\prime}$ such that $B_{Q}^{\prime}$ becomes an algebra. If $f, f^{\prime} \in S(Q)$ are simple functions $f=\sum_{i=1}^{n} f\left(q_{i}\right) \eta_{q_{i}}, f^{\prime}=\sum_{i=1}^{n^{\prime}} f^{\prime}\left(q_{i}^{\prime}\right) \eta_{q_{i}^{\prime}}$, define $f \cdot f^{\prime}=\sum_{i=1}^{n} \sum_{k=1}^{n^{\prime}} f\left(q_{i}\right) \cdot f^{\prime}\left(q_{k}^{\prime}\right) \eta_{q_{i} \wedge q_{k}^{\prime}}$. By this definition $S(Q)$ is a commutative algebra and

$$
\left\|f \cdot f^{\prime}\right\|=\sup _{i, k}\left(\left|f\left(q_{i}\right)\right| \cdot\left|f^{\prime}\left(q_{k}^{\prime}\right)\right|\right) \leqq\|f\| \cdot\left\|f^{\prime}\right\|
$$

shows that the multiplication can be extended to $B_{Q}^{\prime}$, Moreover $f \in \hat{L}_{Q}$, $f^{\prime} \in \hat{L}_{Q}$ implies $f \cdot f^{\prime} \in \hat{L}_{Q}$.

Proof of Theorem 8. Let $A_{M}$ denote the free Boolean ring on $M=\hat{L}_{Q}$ ([5,6], Appendix to this paper). Every element $a \in A_{M}, a \neq 0,1$, has a unique representation

$$
a=\bigvee_{\sigma=1}^{m}\left(\bigwedge_{i=1}^{n} p_{f_{i}}^{-1}\left(\delta_{i \sigma}\right)\right) \quad f_{i} \in \hat{L}_{Q}, \delta_{i \sigma}=0,1
$$


by a union of monomials with the same base such that all monomials are different (thus disjoint) and the base is minimal.

Define an $\hat{L}_{Q}$-valued measure $f(a)$ on $A_{M}$ as follows:

$$
f(a)=\sum_{\sigma=1}^{m}\left(\prod_{i=1}^{n} \delta_{i \sigma}\left(f_{i}\right)\right)
$$

if $a$ has the representation (11). Here $\Pi$ denotes the product introduced above and

$$
\delta_{i \sigma}\left(f_{i}\right)= \begin{cases}f_{i} & \text { if } \delta_{i \sigma}=1 \\ \left(1-f_{i}\right) & \text { if } \delta_{i \sigma}=0 .\end{cases}
$$

Since $f(a)=f(a) \cdot f^{\prime}+f(a) \cdot\left(1-f^{\prime}\right), f^{\prime} \in \hat{L}_{Q}$, it is easily seen that (12) still holds if the base of the monomials of the representation (11) is extended corresponding to Eqs. (16), (17) in the appendix, and the base is no longer minimal. The additivity of $f(a)$ now is an immediate consequence of the representation of $q_{1} \vee q_{2}$ (if $q_{1} \wedge q_{2}=0$ ) given in the appendix.

To prove that $\hat{L}_{Q}$ is contained in the range of $f(a)$, let $f_{0} \in \hat{L}_{Q}$. $a_{f_{0}}=p_{f_{0}}^{-1}(1)$ is a monomial of $A_{M}$, such that $f\left(a_{f_{0}}\right)=f_{0}$.

The ideal of elements $a \in A_{M}$ of measure zero is

$J_{0}=\left\{a \in A_{M} / a=0\right.$ or $a$ has representation (11) and $\prod_{i=1}^{n} \delta_{i \sigma}\left(f_{i}\right)=0$ for all $\left.\sigma\right\}$.

$Q^{\prime}=A_{M} / J_{0}$ denotes the quotient ring and $\varphi$ the canonical homomorphism $\varphi: A_{M} \rightarrow A_{M} / J_{0}=Q^{\prime}$.

Without altering the range of $f(a), f^{\prime}(\varphi(a))=f(a)$ defines an effective $\hat{L}_{Q^{-}}$alued measure on $Q^{\prime}$.

Now it will be shown that there is an injective homomorphism $j: Q \rightarrow Q^{\prime}$ such that $Q$ may be identified with a subring of $Q^{\prime}$ and $f^{\prime}(j(q))=\eta_{q}$ if $q \in Q$. Thus $f^{\prime}(a), a \in Q^{\prime}$, is an extension of the $\hat{L}_{Q^{-}}$-valued measure $q \mapsto \eta_{q}$.

If $q \in Q, a_{q}=p_{\eta_{q}}^{-1}$ (1) is a monomial in $A_{M}$. Define $j(q)=\varphi\left(a_{q}\right)$. To prove $j\left(q^{*}\right)=(j(q))^{*}$ it is sufficient to show $f\left(a_{q^{*}}+\left(a_{q}\right)^{*}\right)=0$

$$
\begin{aligned}
a_{q^{*}}+\left(a_{q}\right)^{*} & =p_{q^{*}}^{-1}(1)+p_{q}^{-1}(0) \\
& =\left(p_{q^{*}}^{-1}(1) \wedge p_{q}^{-1}(1)\right) \\
& \vee\left(p_{q^{*}}^{-1}(0) \wedge p_{q}^{-1}(0)\right) .
\end{aligned}
$$

Thus $f\left(a_{q^{*}}+\left(a_{q}\right)^{*}\right)=\eta_{q^{*}} \cdot \eta_{q}+\left(1-\eta_{q^{*}}\right) \cdot\left(1-\eta_{q}\right)=0$. 
To prove $j\left(q_{1} \wedge q_{2}\right)=j\left(q_{1}\right) \wedge j\left(q_{2}\right)$ is sufficient to show $f\left(\left(a_{q_{1}} \wedge a_{q_{2}}\right)\right.$ $\left.+a_{\left(q_{1} \wedge q_{2}\right)}\right)=0$

$$
\begin{aligned}
& \left(a_{q_{1}} \wedge a_{q_{2}}\right)+a_{\left(q_{1} \wedge q_{2}\right)}=\left(\left(p_{q_{1}}^{-1}(1) \wedge p_{q_{2}}^{-1}(1)\right) \wedge p_{q_{1} \wedge q_{2}}^{-1}(0)\right) \\
& \vee\left(\left(p_{q_{1}}^{-1}(0) \vee p_{q_{2}}^{-1}(0)\right) \wedge p_{q_{1} \wedge q_{2}}^{-1}(1)\right) \\
& =\left(p_{q_{1}}^{-1}(1) \wedge p_{q_{2}}^{-1}(1) \wedge p_{q_{1} \wedge q_{2}}^{-1}(0)\right) \\
& \vee\left(p_{q_{1}}^{-1}(0) \wedge p_{q_{2}}^{-1}(0) \wedge p_{q_{1} \wedge q_{2}}^{-1}(1)\right) \\
& \vee\left(p_{q_{1}}^{-1}(0) \wedge p_{q_{2}}^{-1}(1) \wedge p_{q_{1} \wedge q_{2}}^{-1}(1)\right) \\
& \vee\left(p_{q_{1}}^{-1}(1) \wedge p_{q_{2}}^{-1}(0) \wedge p_{q_{1} \wedge q_{2}}^{-1}(1)\right), \\
& f\left(\left(a_{q_{1}} \wedge a_{q_{2}}\right)+a_{q_{1} \wedge q_{2}}\right)=\eta_{q_{1}} \cdot \eta_{q_{2}} \cdot\left(1-\eta_{q_{1} \wedge q_{2}}\right) \\
& +\left(1-\eta_{q_{1}}\right) \cdot\left(1-\eta_{q_{2}}\right) \cdot \eta_{q_{1} \wedge q_{2}} \\
& +\left(1-\eta_{q_{1}}\right) \cdot \eta_{q_{2}} \cdot \eta_{q_{1} \wedge q_{2}} \\
& +\eta_{q_{1}} \cdot\left(1-\eta_{q_{2}}\right) \cdot \eta_{q_{1} \wedge q_{2}}=0 \text {. }
\end{aligned}
$$

Clearly $j$ is injective and $f^{\prime}(j(q))=f^{\prime}\left(\varphi\left(a_{q}\right)\right)=f\left(a_{q}\right)=\eta_{q}$.

Corollary 1. The set $\hat{L}_{Q}$ of classical effects of a classical system described by $\left\langle B_{Q}, B_{Q}^{\prime}\right\rangle$ is a set of coexistent effects.

Corollary 2. Let $Q$ be a Boolean ring and $F(q)$ an effective $\hat{L}$-valued measure on $Q$ such that $F(1)=1$ and $Q$ is $u_{K^{-}}$complete. There is a Boolean ring $Q^{\prime}$ such that $Q$ is a subring of $Q^{\prime}$, the $\hat{L}$-valued measure $F(q)$ can be extended to $Q^{\prime}$ and the range of the extended $\hat{L}$-valued measure consists of the entire convex range $\overline{\mathrm{co}}\{F(q), q \in Q\}$ of $F(q)$.

Thus the convex range of $F(q)$ is a set of coexistent effects.

Proof. There is a continuous linear homomorphism $S^{\prime}: \hat{L}_{Q} \rightarrow \hat{L}$ determined by the $\hat{L}$-valued measure $F(q)$ such that $S^{\prime}\left(\hat{L}_{Q}\right)=\overline{\operatorname{co}}\{F(q)$, $q \in Q\}$ (Theorem 6). Now apply Theorem 8.

A combination of Theorems 1,7 , and 8 gives

Theorem 9. $A$ set $l \subset \hat{L}$ of effects of a quantum mechanical system consists of coexistent effects if $l$ is contained in the convex range of an observable.

\section{Appendix}

\section{Free Boolean Rings}

A Boolean ring $A$ is said to be free on a subset $M$ which generates $A$ if every mapping from $M$ into an arbitrary Boolean ring can be extended to a ring-homomorphism. This ring-homomorphism is unique. It can be shown that for each cardinal number there actually exists a Boolean ring which is free on a set of this cardinality and is unique up to isomorphisms. 
We will not enter into the proof of this statement but sketch the construction of a free Boolean ring on a set $M$. Let $2^{M}$ denote the $M$-fold product space of $\{0,1\} .2^{M}$ is the set of all mappings $f: M \rightarrow\{0,1\}$. If $x \in M, p_{x}$ denotes the canonical projection $p_{x}: 2^{M} \rightarrow\{0,1\}$ and $p_{x}^{-1}(\delta)$ the set of all mappings $f: M \rightarrow\{0,1\}$ which satisfy $f(x)=\delta(\delta=0,1)$. The subsets $p_{x}^{-1}(1), x \in M$, of $2^{M}$ generate a Boolean ring $A_{M}$ of subsets of $2^{M}$. $x \rightarrow p_{x}^{-1}(1)$ is a natural bijection between $M$ and the class $M^{\prime}$ of generators $p_{x}^{-1}(1)$ of $A_{M}$. By means of the duality theorems in the field of Boolean rings it is proved that $A_{M}$ is free on $M^{\prime}([5,6]) . A_{M}$ consists of all subsets of $2^{M}$ which are finite unions of finite intersections of sets $p_{x}^{-1}(\delta)(\delta=0,1)$

$$
A=\left\{a \subset 2^{M} / a=\bigvee_{\sigma=1}^{m}\left(\bigwedge_{i=1}^{n_{\sigma}} p_{x_{i \sigma}}^{-1}\left(\delta_{i \sigma}\right)\right)\right\} .
$$

It is easily seen that

$$
\bigwedge_{i=1}^{n} p_{x i}^{-1}\left(\delta_{i}\right) \neq 0 \text { is equivalent to } x_{i} \neq x_{k} \text { if } \delta_{i} \neq \delta_{k} .
$$

Sets of the form $\bigwedge_{i=1}^{n} p_{x_{i}}^{-1}\left(\delta_{i}\right)$ with $x_{i} \neq x_{k}$ if $i \neq k$ are called monomials and the sets $\left\{x_{1}, \ldots, x_{n}\right\} \subset M$ base of the monomials.

Two monomials with the same base are equal or disjoint. Every element $a \in A_{M}, a \neq 0,1$, has a unique representation

$$
a=\bigvee_{\sigma=1}^{m}\left(\bigwedge_{i=1}^{n} p_{x_{i}}^{-1}\left(\delta_{i \sigma}\right)\right)
$$

by a union of monomials with the same base such that all monomials are different and the base is minimal.

If $a$ has the representation (13) and the base is not minimal there is $x_{i}$ such that for every $\sigma$ exists $\varrho$ with $\delta_{i \sigma} \neq \delta_{i \varrho}$ and $\delta_{k \sigma}=\delta_{k \varrho}$ if $k \neq i$.

Suppose $a_{1}, a_{2} \in A_{M}$ and $a_{1} \wedge a_{2}=0$. We want to give a representation of $a_{1} \vee a_{2}$. Let

$$
\begin{aligned}
& a_{1}=\bigvee_{\sigma=1}^{m_{1}}\left(\bigwedge_{i=1}^{n_{1}} p_{x_{i}}^{-1}\left(\delta_{i \sigma}^{1}\right)\right), \\
& a_{2}=\bigvee_{\sigma=1}^{m_{2}}\left(\bigwedge_{i=1}^{n_{2}} p_{y_{1}}^{-1}\left(\delta_{i \sigma}^{2}\right)\right) .
\end{aligned}
$$

Without loss of generality aśsume

$$
x_{1}=y_{1}, \ldots, x_{\bar{n}}=y_{\bar{n}} \text { and } x_{i} \neq y_{k} \text { if } \bar{n}+1 \leqq i \leqq n_{1} \text { and } \bar{n}+1 \leqq k \leqq n_{2} .
$$


Now (14) and (15) can be written

$$
\begin{aligned}
& a_{1}=\bigvee_{\sigma=1}^{m_{1}} \bigvee_{\delta_{k}=0,1}\left(\bigwedge_{i=1}^{n_{1}} \bigwedge_{k=\bar{n}+1}^{n_{2}}\left(p_{x_{i}}^{-1}\left(\delta_{i \sigma}^{1}\right) \wedge p_{y_{k}}^{-1}\left(\delta_{k}\right)\right)\right), \\
& a_{2}=\bigvee_{\sigma=1}^{m_{2}} \bigvee_{\delta_{k}=0,1}\left(\bigwedge_{i=1}^{n_{2}} \bigwedge_{k=\bar{n}+1}^{n_{1}}\left(p_{y_{i}}^{-1}\left(\delta_{i \sigma}^{2}\right) \wedge p_{x_{k}}^{-1}\left(\delta_{k}\right)\right)\right) .
\end{aligned}
$$

(16) and (17) are of the form

$$
\begin{aligned}
& a_{1}=\bigvee_{\sigma=1}^{m_{1}^{\prime}}\left(\bigwedge_{i=1}^{n_{3}} p_{z_{i}}^{-1}\left(\delta_{i \sigma}^{\prime 1}\right)\right), \\
& a_{2}=\bigvee_{\sigma=1}^{m_{2}^{\prime}}\left(\bigwedge_{i=1}^{n_{3}} p_{z_{i}}^{-1}\left(\delta_{i \sigma}^{\prime 2}\right)\right) .
\end{aligned}
$$

Thus it is possible to extend the base of the monomials given in (14), (15) such that $a_{1}$ and $a_{2}$ have a representation by unions of monomials of a common base. Of course this base is no longer minimal. $a_{1} \wedge a_{2}=0$ implies that all monomials of $a_{1}$ are different from the monomials of $a_{2}$ and $a_{1} \vee a_{2}$ has a representation by the union of all monomials of (18) and (19).

$$
a_{1} \vee a_{2}=\bigvee_{\sigma=1}^{m_{1}^{\prime}}\left(\bigwedge_{i=1}^{n_{3}} p_{z_{i}}^{-1}\left(\delta_{i \sigma}^{\prime 1}\right)\right) \vee \bigvee_{\sigma=1}^{m_{2}^{\prime}}\left(\bigwedge_{i=1}^{n_{3}} p_{z_{i}}^{-1}\left(\delta_{i \sigma}^{\prime 2}\right)\right)
$$

Also in this representation of $a_{1} \vee a_{2}$ the base $\left\{z_{1}, \ldots, z_{n_{3}}\right\}$ is not necessarily minimal.

Acknowledgement. I wish to thank Prof. Dr. G. Ludwig for many valuable suggestions and for his continuous interest in this work.

\section{References}

1. Ludwig, G.: Deutung des Begriffs „physikalische Theorie" und axiomatische Grundlegung der Hilbertraumstruktur der Quantenmechanik durch Hauptsätze des Messens. Lecture Notes in Physics, Vol. 4, Berlin-Heidelberg-New York: Springer 1970

2. Nikodym,O.M.: The mathematical apparatus for quantum mechanics. Grundlehren der Mathematischen Wissenschaften 129. Berlin-Heidelberg-New York: Springer 1966.

3. Kappos, D.A.: Strukturtheorie der Wahrscheinlichkeitsfelder und -räume. Ergebnisse der Mathematik und ihrer Grenzgebiete 24. Berlin-Göttingen-Heidelberg: Springer 1960.

4. Ludwig, G.: Transformations of ensembles and effects. (to be published).

5. Sikorski,R.: Boolean algebras. Berlin-Göttingen-Heidelberg-New York: Springer 1964.

6. Halmos, P. R.: Lectures on Boolean algebras. Toronto-New York- London: Van Norstrand 1966.

H. Neumann

Institut für Theoretische Physik (I)

Universität Marburg

BRD-3550 Marburg/Lahn, Renthof 7

Germany 